CERN-TH.6051/91

UGVA-DPT 1191/03-712

\title{
Improved Bounds on Extended Gauge Models from New LEP Data+
}

\author{
G.Altarelli \\ CERN- Geneva \\ R.Casalbuoni \\ Dipto di Fisica. Università di Firenze \\ INFN Sezione di Firenze \\ S.De Curtis \\ INFN Sezione di Firenze \\ N.Di Bartolomeo, F.Feruglio* and R.Gatto \\ Dépt. de Phys. Théor., Université de Genève
}

\begin{abstract}
The LEP results from last year's run are shown to imply a considerable improvement in the bounds on the possible admixture of a new nonstandard neutral gauge boson in the observed $Z$ state. One finds that the recent results on lepton asymmetries are particularly important in this respect. The cases of extended gauge models based on $\mathrm{E}_{6}$ (for arbitrary orientation in group space of the new U(1) generator) and of left-right models are discussed in detail. We also consider a number of more particular models with specified Higgs structure, where limits on mZ can also be derived.
\end{abstract}

+ Partially supported by the Swiss National Foundation

* On leave from Università di Padova

CERN-TH.6051/91

UGVA-DPT 1191/03-712

March 1991 


\section{INTRODUCTION}

In this note we shall present an analysis of the most recent LEP data in the framework of extended gauge models. The results of the 1990 runs at LEP have now become available. The implications for extended gauge models of these new data on hadronic and leptonic partial widths and on asymmetries at the peak are very constraining and make an update of previous analyses [1], [2] worthwhile.

The extended gauge models that we shall discuss in detail are the left-right model (LR) [3] and a set of models with an extra $U(1)$ contained in $E_{6}$ for arbitrary defining angle $\theta_{2}$ of the corresponding generator in $E_{6}$ space [4]. Most of the results that we derive do not depend on assumptions on the detailed structure of the Higgs sector of the models. However, in some cases, for a number of particular schemes that have been explicitly considered in the literature, we describe additional constraints that follow for the specific structure of the Higgs sector of each model.

In our analysis the standard model radiative corrections to the widths are taken into account at the full one-loop level.

\section{WIDTHS AND ASYMMETRIES IN EXTENDED GAUGE MODELS}

At LEP1 the existence of an extended gauge sector can only cause departures from the standard predictions to the extent that the observed mass eigenstate $\mathrm{Z}$ is in general a mixture of the standard gauge boson $Z_{S}$ and of a new one $Z_{N}$. Given the $Z_{N}$ couplings as specified in each model (e.g. as functions of $\theta_{2}$ ), the effects of such a mixing are completely described in terms of two parameters: the mixing angle $\xi_{0}$ and the shift $\Delta \rho_{M}$ in the $\rho$ parameter due to the mixing. These two parameters are independent unless the Higgs structure of the model is specified. In this case it is possible that the mass of the heavy physical vector boson $Z^{\prime}$ is fixed as well as the relation between $\xi_{0}$ and $\Delta \rho_{M}$.

In models with an extra $\mathrm{U}(1)$, at tree level, before symmetry breaking, $Z_{S}$ and $Z_{N}$ are the states which are coupled to the ordinary neutral current $\mathrm{J}_{3 \mathrm{~L}}-\sin ^{2} \theta \mathrm{W} \mathrm{J}_{\mathrm{em}}$ and to the additional current $\mathrm{J}_{\mathrm{N}}$, respectively:

$$
\mathrm{L}_{\mathrm{NC}}=\frac{\mathrm{e}}{\sin \theta \mathrm{W} \cos \theta \mathrm{W}} \mathrm{Z}_{\mathrm{S}}\left(\mathrm{J}_{3 \mathrm{~L}}-\sin ^{2} \theta \mathrm{W} \mathrm{J}_{\mathrm{em}}\right)+\mathrm{gN}_{\mathrm{N}} \mathrm{Z}_{\mathrm{N}} \mathrm{J}_{\mathrm{N}}
$$


Clearly, $Z_{S}$ has couplings which are formally identical to the neutral vector boson of the standard model as functions of an angle $\theta \mathrm{W}$ whose relation to the basic input parameters of the theory is in general nonstandard and will be specified in the following.

After symmetry breaking $Z_{S}$ and $Z_{N}$ are mixed. The physical vector bosons $\mathrm{Z}$ and $\mathrm{Z}^{\prime}$ :

$$
\begin{aligned}
& Z=\cos \xi_{0} Z_{S}+\sin \xi_{0} Z_{N} \\
& Z^{\prime}=-\sin \xi_{0} Z_{S}+\cos \xi_{0} Z_{N}
\end{aligned}
$$

are mass eigenstates with masses $\mathrm{mZ}_{\mathrm{Z}}$ and $\mathrm{mZ}^{\prime}$. The standard model relation between $\mathrm{mW}$ and $\mathrm{mZ}_{\mathrm{Z}}$ is modified at tree level according to the equation:

$$
\frac{\mathrm{m}_{\mathrm{W}}^{2}}{\mathrm{~m}_{\mathrm{Z}}^{2} \cos ^{2} \theta \mathrm{W}}=\rho_{0}
$$

In Eq. (3) one has:

$$
\rho_{0}=1+\Delta \rho_{M}+\Delta \rho_{S B}
$$

where:

$$
\Delta \rho_{M}=\left[\left(\frac{m Z^{\prime}}{m Z}\right)^{2}-1\right] \sin ^{2} \xi_{0}>0
$$

$\Delta \rho_{M}$ arises because the $\mathrm{Z}$ mass in the absence of mixing is always larger than the observed $Z$ mass: $m_{Z_{S}}^{2}=\left(1+\Delta \rho_{M}\right) m_{Z}^{2}$ (the lowest energy level is pushed down by the perturbation). For LR models there is in general an additional contribution to $\Delta \rho_{M}$ from the charged sector (which is negligible if the new charged vector bosons are much heavier than the neutral $\left.Z^{\prime}\right) . \Delta \rho S B$ stands for a possible contribution from the symmetry breaking (SB) sector of the theory. For example, $\Delta \rho_{S B}$ could arise from the presence of non-doublet Higgses. Unless explicitly stated, in the following we shall always assume that $\Delta \rho_{S B}=0$ at tree level.

Although we have used complete one-loop radiative corrections for the standard model prediction, for simplicity we will refer explicitly to the improved Born approximation [5], which correctly takes into account 


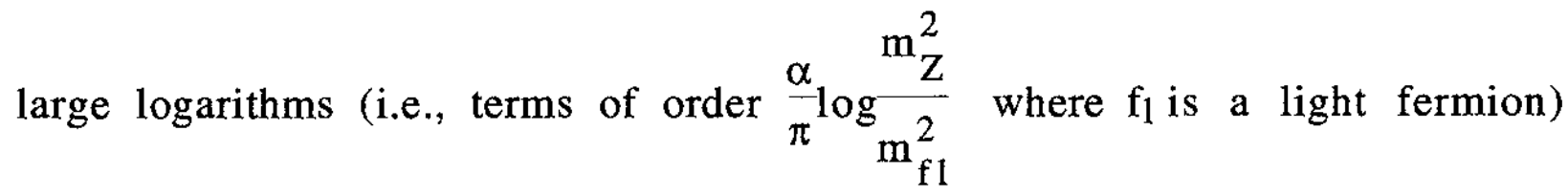
and quadratic terms in the top mass (i.e. terms of order $G_{F m}{ }_{t}^{2}$ ). Loop effects due to the heavy gauge boson $Z^{\prime}$ are generally small for the relevant range of parameters and will be neglected here.

In the improved Born approximation the partial widths $\Gamma(\mathrm{Z}->\mathrm{f} \overline{\mathrm{f}})$ and the forward-backward asymmetry for the fermion $f$ at $\sqrt{\mathrm{s}}=\mathrm{m}_{\mathrm{Z}}$ are given by $(f \neq b)[5]$ :

$$
\begin{aligned}
& \Gamma(\mathrm{Z}-\mathrm{fff})=\frac{\mathrm{G}_{F m^{3}}}{6 \pi \sqrt{2}} \rho \mathrm{N}_{\mathrm{c}}\left[\left(\mathrm{v}_{\mathrm{eff}}^{\mathrm{f}}\right)^{2}+\left(\mathrm{a}_{\mathrm{eff}}^{\mathrm{f}}\right)^{2}\right] \\
& v_{\text {eff }}^{e} a_{\text {eff }}^{e} \quad v_{\text {eff }}^{f}{ }^{a_{e f f}} \\
& A_{F B}=3 \overline{\left(v_{e f f}^{e}\right)^{2}+\left(a_{e f f}^{e}\right)^{2}} \frac{f}{\left(v_{e f f}\right)^{2}+\left(a_{e f f}^{f}\right)^{2}}
\end{aligned}
$$

Other asymmetries (e.g., the $\tau$ polarisation asymmetry) can similarly be expressed in terms of $v_{\text {eff }}^{f} \underset{\text { eff }}{f}$. Here $N_{c}=3\left(1+\delta_{Q C D}\right)$ for quarks, $N_{c}=1$ for leptons, and $\rho$ also includes the large radiative corrections arising from the top quark loop as well as the logarithmic terms from the Higgs (included in $\Delta \rho S B$ )

$$
\rho=1+\Delta \rho_{M}+\Delta \rho_{S B}+\Delta \rho_{t}=1+\Delta \rho
$$

where $\Delta \rho_{t} \cong \frac{3 G_{F m}{ }_{t}^{2}}{8 \pi^{2} \sqrt{2}}+\ldots \ldots$. The effective vector and axial vector couplings $v_{\text {eff }}^{f}$ and $a_{\text {eff }}^{f}$ are given by a superposition of the corresponding $Z_{S}$ and $\mathrm{Z}_{\mathrm{N}}$ couplings: 


$$
v_{\text {eff }}^{f}=\cos \xi_{0} v_{S}^{f}+\sin \xi_{0} v_{N}^{f} ; \quad \underset{a_{e f f}}{f}=\cos \xi_{0} \underset{S}{f}+\sin \xi_{0} a_{N}^{f}
$$

In particular the $Z_{S}$ couplings are given by:

$$
\mathrm{v}_{S}^{\mathrm{f}}=\mathrm{T}_{3 \mathrm{~L}}^{\mathrm{f}}-2 \overline{\bar{s}}_{\mathrm{W}}^{2} Q^{\mathrm{f}} ; \quad \mathrm{a}_{S}^{\mathrm{f}}=-\mathrm{T}_{3 \mathrm{~L}}^{\mathrm{f}}
$$

where $T_{3 L}^{f}$ and $Q^{f}$ are the $3 r d$ component of the weak isospin and the electric charge of the fermion $\mathrm{f}$. The quantity $\overline{\mathrm{s}}_{\mathrm{W}}^{2}$ given by the relation:

$$
\overline{\mathrm{s}}_{\mathrm{W}}^{2} \overline{\mathrm{c}}_{\mathrm{W}}^{2}=\frac{\pi \alpha\left(\mathrm{m}_{\mathrm{Z}}\right)}{\sqrt{2} \mathrm{G}_{\mathrm{FmZ}} \mathrm{m}^{2} \rho}
$$

is the effective $\sin ^{2} \theta \mathrm{W}$ for the on-shell $\mathrm{Z}$ couplings. Here $\alpha\left(\mathrm{m}_{\mathrm{Z}}\right)=\alpha /(1-\Delta \alpha)$ × $1 / 128.8$. Because of the effect of $\Delta \rho_{M}$, it differs from the corresponding quantity $\mathrm{s}_{\mathrm{W}}$ in the standard model, for fixed input parameter $\alpha, \mathrm{G}_{\mathrm{F}}, \mathrm{m}_{\mathrm{Z}}$, $\mathrm{m}_{\mathrm{f}}, \mathrm{m}_{\mathrm{H}}$, according to the relation:

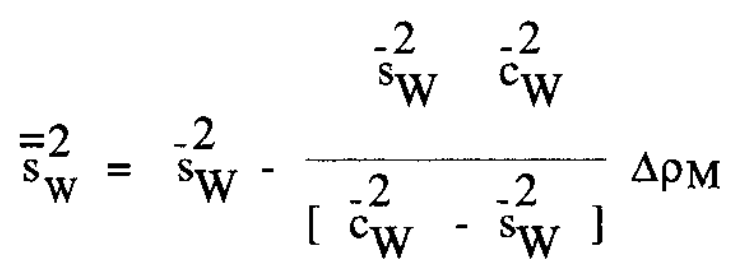

Note that the case $\Gamma(Z->b \bar{b})$ is exceptional because, as is well known, there are additional large terms of order $G_{F m}{ }_{t}^{2}$ from vertex diagrams, which are taken into account [5],[6] by replacing $1+\Delta \rho_{\mathrm{t}}$ in $\rho$ by $1-\frac{1}{3} \Delta \rho_{\mathrm{t}}$ and $\overline{\mathrm{s}}_{\mathrm{W}}^{2}$ by $\overline{\mathrm{s}}_{\mathrm{W}}^{2}\left(1+\frac{2}{3} \Delta \rho_{\mathrm{t}}\right)$ in Eqs. (6) and (10). The vector and axial vector couplings $v_{N}^{f}$ and $a_{N}^{f}$ of the new vector boson $Z_{N}$ depend on the model. 
For the LR and the $E_{6}$ models that we consider here, $v_{N}$ and $a_{N}$ are collected in Table 1. The entries in Table 1 correspond to $\mathrm{gN}_{\mathrm{N}}$ [defined in Eq.(1)] given by $\mathrm{g}_{\mathrm{N}}=\mathrm{g}^{\prime}=\mathrm{g} \tan \theta \mathrm{W}$. This identification is clearly not necessary, but should be valid as an order of magnitude if grand unification occurs at a large scale.

It is useful to consider the deviations of the predicted $Z$ partial widths and asymmetries from the corresponding standard model values, linearised in $\xi_{0}$ and $\Delta \rho_{M}$. As an example, for $\Gamma(\mathrm{Z}->\mathrm{ff})$ one obtains, to first order in $\xi_{0}$ :

$$
\delta \Gamma_{\mathrm{f}}=\frac{\mathrm{G}_{\mathrm{Fm}} \mathrm{Z}_{\mathrm{Z}}^{3}}{24 \pi \sqrt{2}}\left(\mathrm{~A}_{\mathrm{f}} \Delta \rho_{\mathrm{M}}+\mathrm{B}_{\mathrm{f}} \xi_{0}\right)
$$

In Eq.(13) the coefficients $A_{f}$ are uniquely determined in terms of the standard model parameters, whereas the $B_{f}$ also depend on the couplings $v_{N}$ and $a_{N}$. Their expressions follow immediately from Eqs.(6)(12) together with Table 1 and can be found in Ref. [1].

We have seen that at the $\mathrm{Z}$ peak all effects of extended gauge models are given in terms of $\Delta \rho_{\mathrm{M}}$ and $\xi_{0}$, which are independent if the mass $\mathrm{mZ}^{\prime}$ of the heavy vector boson $Z^{\prime}$, which appears in Eq. (5), is not known. We now discuss the constraints imposed by the data on $\Delta \rho_{M}$ and $\xi_{0}$ in $E_{6}$ models for all values of $\theta_{2}$, and in LR models.

\section{THE DATA AND THEIR IMPLICATIONS}

The data on the widths and asymmetries that we use in the present analysis of extended gauge models, together with the values of $v_{\text {eff }}$ and $\mathrm{a}_{\text {eff }}^{\mathrm{f}}$ as obtained by the LEP experiments, are collected in Table 2 [7]. In the following the $\mathrm{Z}$ mass was fixed at the value $\mathrm{mZ}=91.174 \mathrm{GeV}$.

The $\chi^{2}$ function minimized in the fit is the sum of 4 terms: the total width $\Gamma_{\mathrm{Z}}$, the hadronic width $\Gamma_{\mathrm{h}}$, the leptonic width $\Gamma_{1}$ and the ratio 
$\left(v_{\text {eff }}^{e} / a_{\text {eff }}^{e}\right)^{2}$. The theoretical expectation for the widths has been defined

as the sum of the full one-loop standard model prediction plus the corresponding deviation as given by Eq. (13). For $\Gamma_{Z}$ and $\Gamma_{h}$ the QCD correction was evaluated for $\alpha_{s}\left(m_{Z}\right)$ in the range of $0.11-0.13$ which is selected by the results of the measurements of $\alpha_{s}$ at LEP [9].

The resulting constraints on $\Delta \rho_{M}$ and $\xi_{0}$ for $E_{6}$ models are plotted in Figs. 1 and 2 for all values of $\theta_{2}$.

In Fig. 1 the $1.64 \sigma$ upper and lower limits on $\Delta \rho_{M}$ are displayed for $\mathrm{m}_{\mathrm{t}}=\mathrm{m}_{\mathrm{H}}=100 \mathrm{GeV}$ and for the two values of $\alpha_{\mathrm{s}}\left(\mathrm{m}_{\mathrm{Z}}\right), 0.11$ and 0.13 . Varying $\mathrm{m}_{\mathrm{t}}$ and $\mathrm{m}_{\mathrm{H}}$, the allowed region is shifted along the vertical direction. It is possible to see that the maximum (with respect to $\theta_{2}$ ) of the upper limits varies according to the following relation:

$$
\Delta \rho_{M}<0.01-\Delta \rho_{S B}-\Delta \rho_{t}
$$

where $\Delta \rho_{\text {SB }}$ and $\Delta \rho_{\mathrm{t}}$ are the standard model Higgs and top contributions to $\Delta \rho$ and we have taken $\alpha_{s}\left(m_{z}\right)=0.11$ so as to obtain the most conservative upper bound. Therefore we can express the above result as an upper limit on $\Delta \rho$ [see Eq. (8)] which is $\mathrm{m}_{\mathrm{t}^{-}}$and $\mathrm{m}_{\mathrm{H}}$-independent: $\Delta \rho$ $<0.01$. Note that this bound from LEP data is better than the one obtained from $\mathrm{mZ}$ and the recent data from $\mathrm{CDF}$ and $\mathrm{UA} 2$ on $\mathrm{mw} / \mathrm{mz}$, which is $\Delta \rho<0.014$ [8]. Note that while $\Delta \rho_{M}>0$ in models with an extra $\mathrm{U}(1)$ [Eq. (5)] we also allowed negative values for $\Delta \rho_{M}$ in Fig. 1. This is not without interest, because we assumed $\Delta \rho \mathrm{SB}=0$ at tree level and this assumption could be wrong. Thus one can reinterpret Fig. 1 as limits on $\Delta \rho_{M}+\Delta \rho_{S B}$. Clearly the upper limit on $\Delta \rho_{M}$ is sensitive to $m_{t}$, and there is less space for $\Delta \rho_{M}$ as $m_{t}$ increases. The present constraints are powerful enough, and the allowed values of $\Delta \rho_{M}$ so narrow, that the bounds are also sensitive to sufficiently large variations of $\mathrm{m}_{\mathrm{H}}$. For large $\mathrm{m}_{\mathrm{H}}$ the upper bound on $\Delta \rho_{M}$ is somewhat relaxed.

In Fig. 2 the $1.64 \sigma$ allowed band for $\xi_{0}$ is displayed for $\mathrm{m}_{\mathrm{t}}=\mathrm{m}_{\mathrm{H}}=100$ $\mathrm{GeV}$, again for $\alpha_{\mathrm{s}}\left(\mathrm{m}_{\mathrm{z}}\right)=0.11$ and 0.13 . We have checked that in this case the bounds are very little affected by variations of $\mathrm{m}_{\mathrm{t}}$ and $\mathrm{m}_{\mathrm{H}}$. If a comparison is made with the now superseded results in Refs. [1],[2] we see that the limits on $\xi_{0}$ have much improved, particularly in the vicinity 
of $\theta_{2}=0$, where the separate measurements of $v_{e f f}^{e}$ and $a_{e f f}^{e}$ are very powerful in limiting $\xi_{0}$.

The corresponding results on the LR model are reported in Table 3 . The same pattern is found also in this case.

\section{SPECIAL MODELS}

We now consider the consequences of restricting the scalar structure of the model to a particular choice. We restrict ourselves to a Higgs structure that obeys the physical requirement of vanishing mixing for large $\mathrm{m}_{\mathrm{Z}}$, i.e. we demand that, for large $\mathrm{m}_{\mathrm{Z}}$ :

$$
\xi_{0}=c\left(\frac{\mathrm{mZ}}{\mathrm{mZ}^{\prime}}\right)^{2}
$$

with $c$ a constant which depends on the Higgs structure and vacuum expectation values [1]. Once $\mathrm{c}$ is given, in a particular model, then either $\xi_{0}$ or $\Delta \rho_{M}$ can be eliminated in favour of $\mathrm{mZ}^{\prime}$. In this case a bound on $\mathrm{mZ}^{\prime}$ is directly obtained from the data. From the following illustrative cases we can appreciate the typical sensitivity of the present LEP data to the heavy vector boson mass.

Figure 3 refers to the so-called minimal left-right model, with Higgs particles transforming as complex $(2,2,0),(3,1,0)$ and $(1,3,0)$ representations of the gauge group $\mathrm{SU}(2)_{\mathrm{L}} \times \mathrm{SU}(2)_{\mathrm{R}} \mathrm{XU}(1)_{\mathrm{B}-\mathrm{L}}$. In this case one has [3] $c=\sqrt{\cos 2 \theta w}=0.73$. Assuming negligible additional mixing from the charged sector and no further exotic contribution to the $\rho$ parameter one finds a lower bound on $\mathrm{m}_{\mathrm{ZR}}$ of about $950(800) \mathrm{GeV}$ for a standard Higgs mass of $100 \mathrm{GeV}(1 \mathrm{TeV})$ and a top mass of $90 \mathrm{GeV}$ (in both cases). The lower bound increases with $\mathrm{m}_{\mathrm{t}}$. For example, taking $\mathrm{m}_{\mathrm{t}}=$ $130 \mathrm{GeV}$, we find a lower bound of about 1400 (1000) GeV.

The models [10] $\chi$ (or B ) and $\eta$ (or A ) are illustrated in Figs 4 and 5 , respectively. The new gauge generator corresponds to $\theta_{2}=52.24^{0}$ in the $\chi$ model and to $\theta_{2}=0$ in the $\eta$ model. For two Higgs doublets one finds [10] $c_{\chi}=-0.39$ and $-0.63<c_{\eta}<-0.24$. One then obtains a lower bound for $\mathrm{m}_{\mathrm{Z}_{\chi}}$ of about $540(500) \mathrm{GeV}$ for $\mathrm{m}_{\mathrm{H}}=100 \mathrm{GeV}(1 \mathrm{TeV})$ with $\mathrm{m}_{\mathrm{t}}=90 \mathrm{GeV}$. For $\mathrm{m}_{\mathrm{t}}=130 \mathrm{GeV}$ this limit becomes $700(570) \mathrm{GeV}$. Less stringent limits are obtained on $\mathrm{mZ \eta}$, corresponding to the smaller absolute value allowed for c. For $\mathrm{m}_{\mathrm{t}}=90 \mathrm{GeV}$ the lower bound is in the range $300-900 \mathrm{GeV}$ 
depending on the value of $c$ in the allowed range and on $m_{H}$ in the interval $0.1-1 \mathrm{TeV}$.

\section{CONCLUSION}

By precisely determining the couplings to fermions of the physical $\mathrm{Z}$, the data collected on the $\mathrm{Z}$ peak at LEP impose severe constraints on the possible amount of mixing of a non-standard neutral vector boson. The effects of such an admixture are in general described by two parameters $\xi_{0}$ and $\Delta \rho_{M}$. We have studied the bounds on $\xi_{0}$ and $\Delta \rho_{M}$ implied by the whole set of LEP data and shown that they have become very stringent. Actually, the impact of LEP data alone is by now more important in this respect than the totality of all previous data on neutral current processes. However, it must be stressed that a new neutral vector boson could in principle have a negligible mixing to the physical $\mathrm{Z}$. In this case its effect would not be detectable at LEP. Yet the contributions of $Z^{\prime}$ exchange could be important for all neutral current processes which do not involve an on-shell $\mathrm{Z}$. For example, clearly the $\mathrm{Z}^{\prime}$ could be produced at hadron colliders of sufficiently large centre-ofmass energy. This complementary character results in powerful overall constraints on extended gauge models. As an example, the present new bounds from LEP have been found to give substantial limitations on possible deviations in atomic parity violation experiments as expected within such models [11]. After completion of this paper we received a work [12] where a similar, less complete analysis, is performed.

\section{ACKNOWLEDGEMENT.}

We would like to thank W.Hollik for providing us with a programme to compute the full one-loop standard model predictions for the widths. 


\section{REFERENCES}

[1] G. Altarelli, R. Casalbuoni, D. Dominici, F. Feruglio and R. Gatto, Mod. Phys. Lett. A5 (1990) 495 and Nucl. Phys. B342 (1990) 15;

G. Altarelli, R. Casalbuoni, F. Feruglio and R. Gatto, Phys. Lett. B 245 (1990) 669.

[2] J. Layssac, F.M. Renard and C. Verzegnassi, LAPP preprint LAPPTH-290/90 (1990);

J.W.F. Valle and M.C. Gonzalez-Garcia, preprint from Valencia, FTUV/900-45 (1990).

[3] J.C. Pati and A. Salam, Phys. Rev. D10 (1974) 275;

R.N. Mohapatra and J.C. Pati, Phys. Rev. D11 (1975) 566, 2559;

G. Senjanovic and R.N. Mohapatra, Phys. Rev. D12 (1975) 152;

G. Senjanovic, Nucl. Phys. B153 (1979) 334.

[4] E. Witten, Nucl. Phys. B258 (1985) 75;

M. Dine, V. Kaplunovsky, M. Mangano, C. Nappi and N. Seiberg, Nucl. Phys. B259 (1985) 519;

S. Cecotti, J.-P. Derendinger, S. Ferrara, L. Girardello and $M$. Roncadelli, Phys. Lett. B156 (1985) 318;

J.D. Breit, B.A. Ovrut and G. Segrè, Phys. Lett. B158 (1985) 33;

E. Cohen, J. Ellis, K. Enqvist and D.V. Nanopoulos, Phys. Lett. B165 (1985) 76;

J. Ellis, K. Enqvist, D.V. Nanopoulos and F. Zwirner, Nucl. Phys. B276 (1986) 14; Mod. Phys. Lett. A1 (1986) 57;

F. Del Aguila, G. Blair, M. Daniel and G.G. Ross, Nucl. Phys. B272 (1986) 413;

L. Ibanez and J. Mas, Nucl. Phys. B286 (1987) 107.

[5] M. Consoli, W. Hollik and F. Jegerlehner, in Z Physics at LEP1, Eds. G. Altarelli, R. Kleiss and C. Verzegnassi, CERN report 89-08 Vol. 1 (CERN, Geneva, 1989), p.7;

G. Altarelli, Proc. XIV Intern. Symp. on Lepton and Photon Interactions (Stanford, CA, 1989);

D.C. Kennedy et al., Nucl. Phys. B321 (1989) 83;

B.W. Lynn, Stanford Univ. preprint SU-ITP-867 (1989);

A. Blondel, CERN preprint CERN-EP/89-04 (1989);

A. Sirlin, CERN preprint CERN-TH.5506 (1989).

[6] A.A. Akhundov, D. Yu.Bardin and T. Riemann, Nucl. Phys. B276 (1988) 1;

F. Diakonos and W. Wetzel, Heidelberg preprint HD-THEP-88-21 (1988); 
W. Beenakker and W. Hollik, Z. Phys. C40 (1988) 141;

J. Bernabeú, A. Pich and A. Santamaria, Phys. Lett. B200 (1988) 569 ;

B.W. Lynn and R.G. Stuart, CERN preprint TH.5786/90 (1990).

[7] The data on $m_{z}$ and the widths were presented by G.J. Bobbink at the XXV Rencontres de Moriond, Les Arcs, France, March 10-17, 1991. The data on $\mathrm{g}^{2} \mathrm{~V}, \mathrm{~g}^{2} \mathrm{~A}$ were reported by P. Ratoff at the same meeting.

[8] See, for example, G. Altarelli, Proceedings of the Neutrino '90 Conference, Geneva, 1990 and CERN preprint TH.5834/90 (1990).

[9] See for example, S. Bethke, Proceedings of the Worskhop on Jet Physics at LEP and HERA, Durham, 1990 and CERN-PPE/91-36 (1991).

[10] G. Costa, J. Ellis, G.L. Fogli, D.V. Nanopoulos and F. Zwirner, Nucl. Phys. B297 (1988) 244l;

U. Amaldi, A. Bohm, L.S. Durkin, P. Langacker A.K. Mann, A. Sirlin and H.M. Williams, Phys. Rev. D36 (1987) 1385.

[11] G. Altarelli, R. Casalbuoni, S. De Curtis, N. Di Bertolomeo, F. Feruglio and R. Gatto, CERN preprint TH.6028/91 (1991).

[12] A. Chiappinelli, Annecy preprint LAPP-TH-327/91 (1991). 


\section{FIGURE CAPTIONS}

Fig. 1: Allowed range (90\% CL) of $\Delta \rho_{M}$ versus $\theta_{2}$ (defining the embedding of the extra $\mathrm{U}(1)$ in $\left.\mathrm{E}_{6}\right)$, for $\mathrm{m}_{\mathrm{top}}=\mathrm{m}_{\mathrm{Higgs}}=$ $100 \mathrm{GeV}$ and $\alpha_{s}=0.11$ (solid) or $\alpha_{s}=0.13$ (dashed).

Fig. 2: Allowed range $(90 \% \mathrm{CL})$ of $\xi_{0}$ versus $\theta_{2}$ (defining the embedding of the $\mathrm{U}(1)$ in $\left.\mathrm{E}_{6}\right)$ for $\mathrm{m}_{\mathrm{top}}=\mathrm{m}_{\mathrm{Higgs}}=100 \mathrm{GeV}$ and $\alpha_{\mathrm{s}}=0.11$ (solid) or $\alpha_{\mathrm{s}}=0.13$ (dashed).

Fig. 3: Lower bound on $\mathrm{m}_{\mathrm{z}^{\prime}}(90 \% \mathrm{CL})$ for the left-right model as a function of $\mathrm{m}_{\mathrm{top}}$, for $\mathrm{m}_{\mathrm{Higgs}}=100 \mathrm{GeV}$ (full line) and $\mathrm{m}_{\mathrm{Higgs}}$ $=1 \mathrm{TeV}$ (dotted line). Mixing effects in the charged sector have been neglected.

Fig. 4: Lower bound on $\mathrm{m}_{\mathrm{z}^{\prime}}(90 \% \mathrm{CL})$ for the $\chi$ model, as a function of $\mathrm{m}_{\text {top }}$, for $\mathrm{m}_{\mathrm{Higgs}}=100 \mathrm{GeV}$ (full line) and $\mathrm{m}_{\mathrm{Higgs}}=1 \mathrm{TeV}$ (dotted line).

Fig. 5: Lower bound on $\mathrm{m}_{\mathrm{z}^{\prime}}(90 \% \mathrm{CL})$ for the $\eta$ model, as a function of $\mathrm{m}_{\text {top }}$, for $\mathrm{m}_{\mathrm{Higgs}}=100 \mathrm{GeV}$ (full lines) and $\mathrm{m}_{\mathrm{Higgs}}=1 \mathrm{TeV}$ (dotted lines). For each Higgs mass, the band within the two lines corresponds to the allowed range for the parameter $c$ defining the proportionality between $\xi_{0}$ and $\left(\mathrm{mZ}_{\mathrm{Z}} / \mathrm{m}_{\mathrm{Z}^{\prime}}\right)^{2}$ [Eq. (15)]. 


\section{Table 1}

Vector and axial couplings of fermions to the unmixed new vector boson $Z_{N}$ in the case of extra U(1) and LR models. For left-right models, we have put in the text $\lambda=g_{L} / g_{R}=1$. As a consequence $y=\sqrt{\cos 2 \theta}$.

$\left.\begin{array}{c}\text { Extra U(1) Models } \\ v_{N}^{u}=0, \\ a_{N}^{u}=-\frac{2}{3} \sin \theta \cos \theta_{2}, \\ v_{N}^{d}=\frac{1}{2} \sin \theta\left(\cos \theta_{2}+\sqrt{\frac{5}{3}} \sin \theta_{2}\right) \\ a_{N}^{d}=\sin \theta\left(-\frac{1}{6} \cos \theta_{2}+\frac{1}{2} \sqrt{\frac{5}{3}} \sin \theta_{2}\right), \\ v_{N}^{e}=-\frac{1}{2} \sin \theta\left(\cos \theta_{2}+\sqrt{\frac{5}{3}} \sin \theta_{2}\right), \\ a_{N}^{e}=a_{N}^{d}, \\ v_{N}^{\nu}=-\sin \theta\left(\frac{1}{6} \cos \theta_{2}+\frac{1}{2} \sqrt{\frac{5}{3}} \sin \theta_{2}\right) \\ a_{N}^{\nu}=-v_{N}^{\nu} . \\ \text { Left-Right Models } \\ v_{N}^{f}=\left[\frac{\cos ^{2} \theta}{\lambda y}\left(T_{3 R}-2 \lambda^{2} \sin ^{2} \theta Q_{e m}\right)+\frac{\lambda \sin ^{2} \theta}{y}\left(T_{3}-2 \sin ^{2} \theta Q_{e m}\right]\right. \\ a_{N}^{f}=\left[\frac{\cos ^{2} \theta}{\lambda y} T_{3 R}-\frac{\lambda \sin ^{2} \theta}{y} T_{3 L}\right]\end{array}\right]$


Table 2

Results of the 1990 LEP run, as presented in Ref. [7]. Note that the reported expressions of $g_{V}^{2}, g_{A}^{2}$ follow from the comparison of Eq. (6) with the definitions adopted in Ref. [7] for the vector and axial vector couplings.

\begin{tabular}{|c|c|c|c|c|c|}
\hline Parameter & ALEPH & DELPHI & L3 & OPAL & Average \\
\hline$M_{Z}(\mathrm{GEV})$ & $91.183 \pm 0.009$ & $91.175 \pm 0.010$ & $91.181 \pm 0.010$ & $91.160 \pm 0.009$ & $91.174 \pm 0.02$ \\
$\Gamma_{Z}(\mathrm{GeV})$ & $2.484 \pm 0.017$ & $2.454 \pm 0.020$ & $2.501 \pm 0.017$ & $2.491 \pm 0.017$ & $2.485 \pm 0.009$ \\
$\Gamma_{\mathrm{had}}(\mathrm{GeV})$ & $1.750 \pm 0.015$ & $1.718 \pm 0.021$ & $1.742 \pm 0.019$ & $1.739 \pm 0.017$ & $1.740 \pm 0.009$ \\
$\Gamma_{\ell}(\mathrm{MeV})$ & $83.35 \pm 0.68$ & $83.4 \pm 0.9$ & $83.6 \pm 0.8$ & $83.0 \pm 0.7$ & $83.31 \pm 0.40$ \\
$g_{a}^{2}=\rho a_{\text {eff }}^{e^{2}}$ & $0.2489 \pm 0.0021$ & $0.250 \pm 0.003$ & $0.250 \pm 0.003$ & $0.2495 \pm 0.0022$ & $0.2495 \pm 0.0012$ \\
$g_{v}^{2}=\rho v_{\text {eff }}^{e^{2}}$ & $0.0016 \pm 0.0006$ & $0.0008 \pm 0.0014$ & $0.0021 \pm 0.0012$ & $0.0006 \pm 0.0007$ & $0.0013 \pm 0.0004$ \\
\hline
\end{tabular}


Table 3

Combined fit of LEP data for the left-right model, with best values $\left(\xi_{0}, \Delta \rho_{M}\right)$ and lower $\left(\xi_{\text {low }}, \Delta \rho_{M \text { low }}\right)$ and upper $\left(\xi_{u p}, \Delta \rho_{M}\right.$ up $)$ bounds $(90 \%$ CL).

\begin{tabular}{|c|c|c|c|c|}
\hline & \multicolumn{2}{|c|}{$m_{t}=100 \mathrm{GeV}$} & \multicolumn{2}{c|}{$m_{t}=150 \mathrm{GeV}$} \\
\hline & $m_{H}=100 \mathrm{GeV}$ & $m_{H}=1000 \mathrm{GeV}$ & $m_{H}=100 \mathrm{GeV}$ & $m_{H}=1000 \mathrm{GeV}$ \\
\hline$\xi_{0}$ & $0.0023 \pm 0.0044$ & $0.0025 \pm 0.0045$ & $0.0026 \pm 0.0044$ & $0.0031 \pm 0.0045$ \\
\hline$\Delta \rho_{M}$ & $0.0015 \pm 0.0026$ & $0.0038 \pm 0.0026$ & $-0.0021 \pm 0.0026$ & $0.0004 \pm 0.0026$ \\
\hline$\xi_{\text {low }}$ & -0.0050 & -0.0048 & -0.0047 & -0.0042 \\
\hline$\xi_{\text {up }}$ & +0.0096 & +0.0099 & +0.0099 & +0.0104 \\
\hline$\Delta \rho_{M \text { low }}$ & -0.0029 & -0.0006 & -0.0064 & -0.0040 \\
\hline$\Delta \rho_{M \text { up }}$ & +0.0058 & +0.0081 & +0.0022 & +0.0047 \\
\hline
\end{tabular}




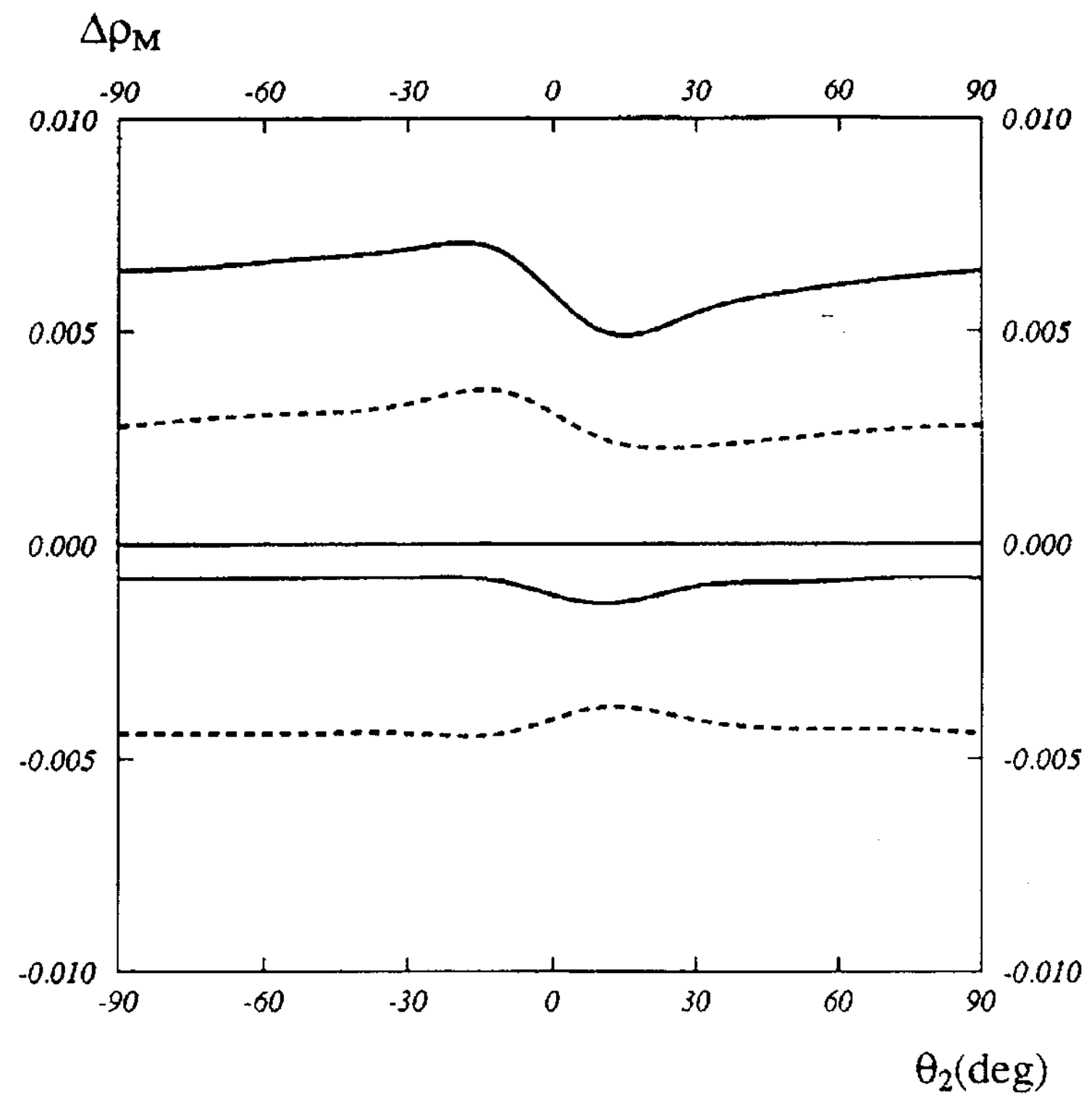

Fig. 1 


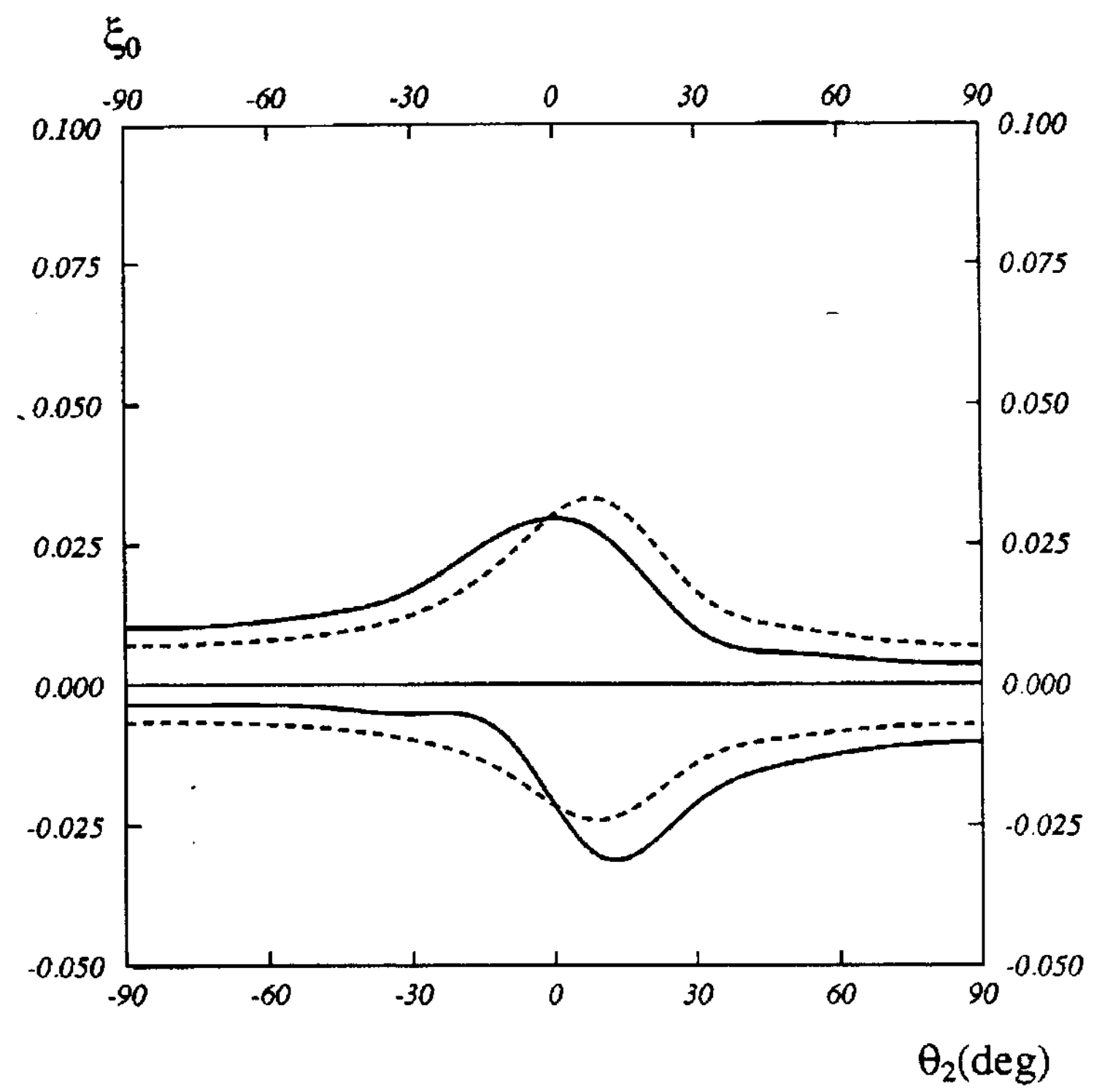

Fig. 2 


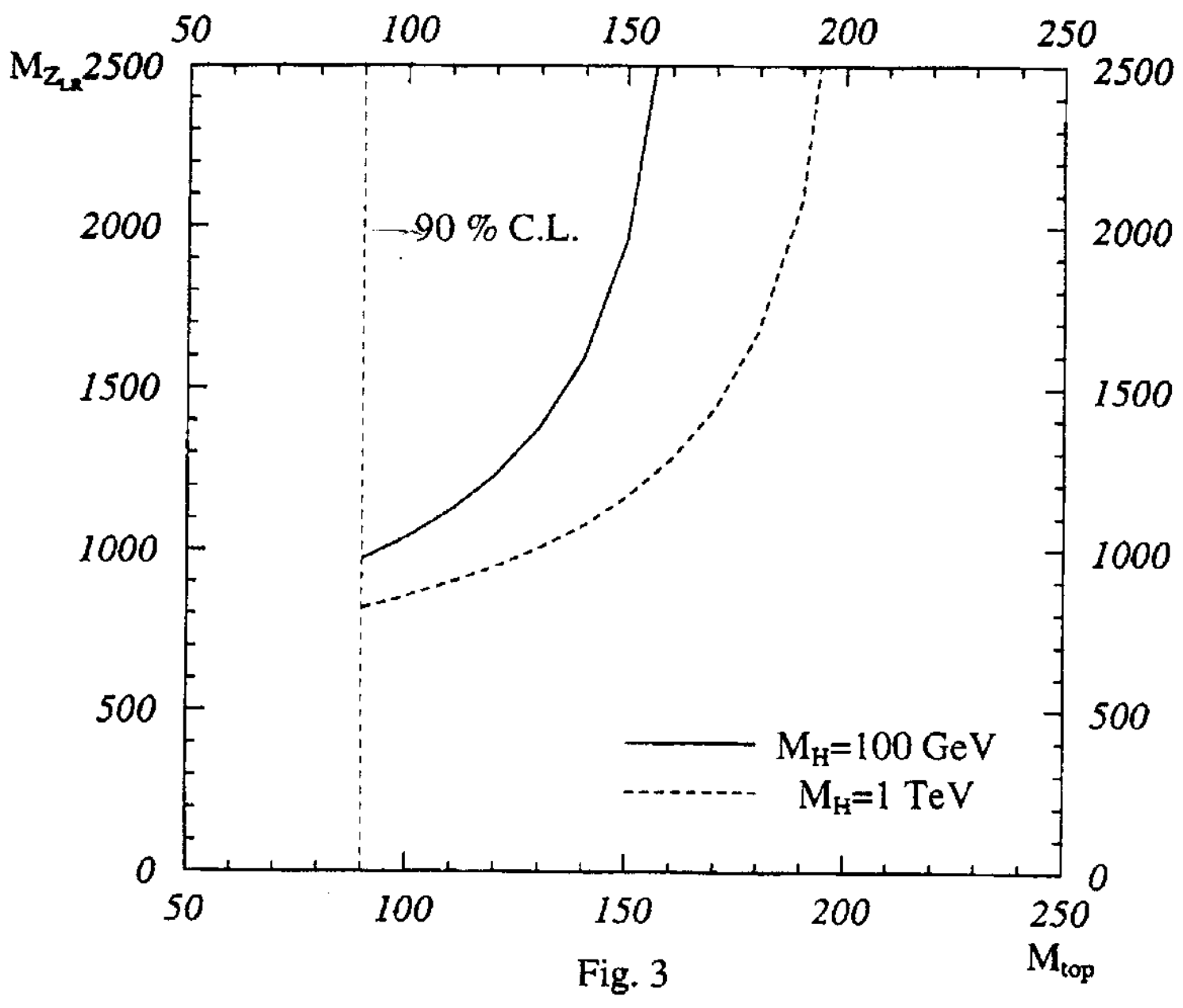




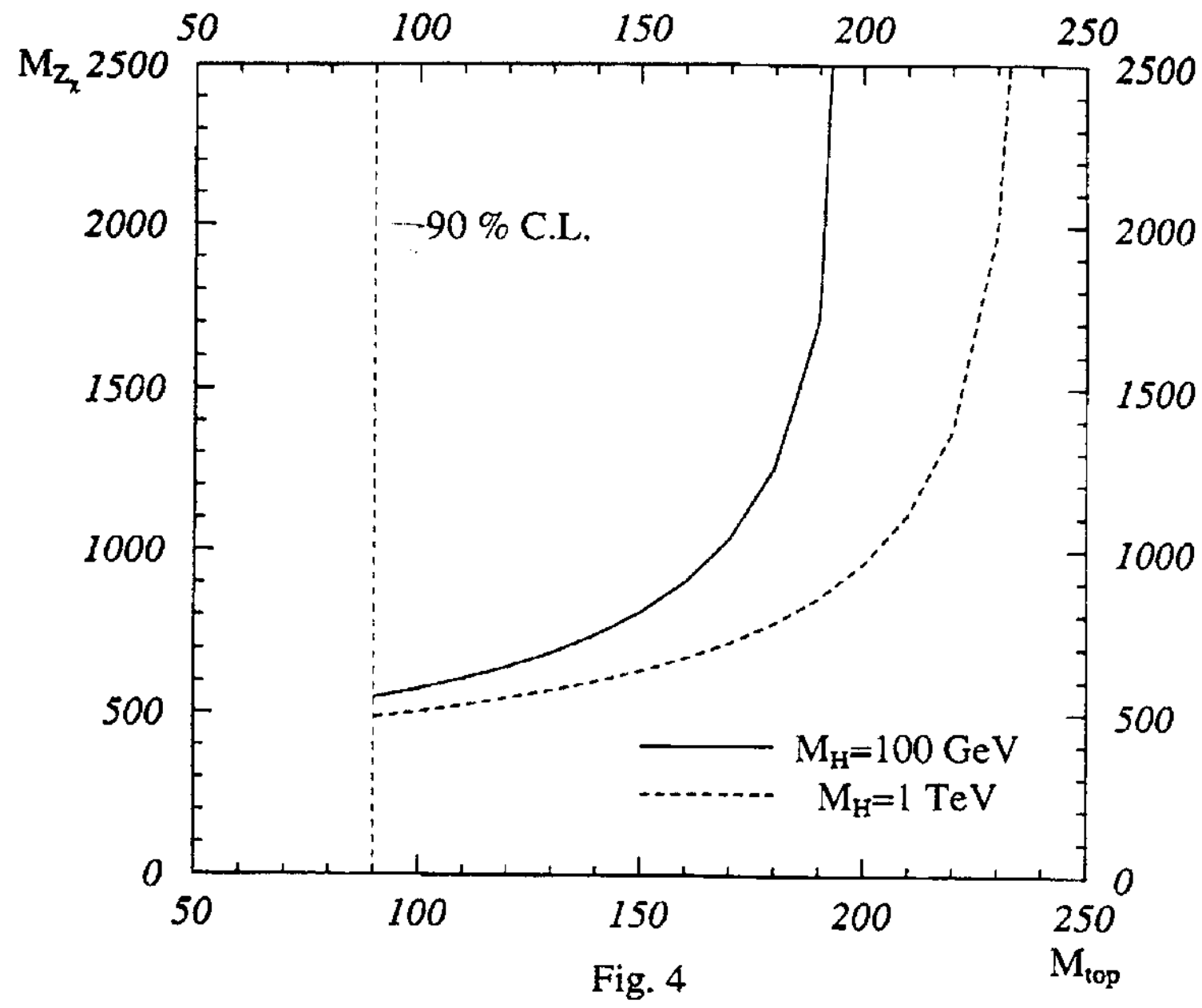




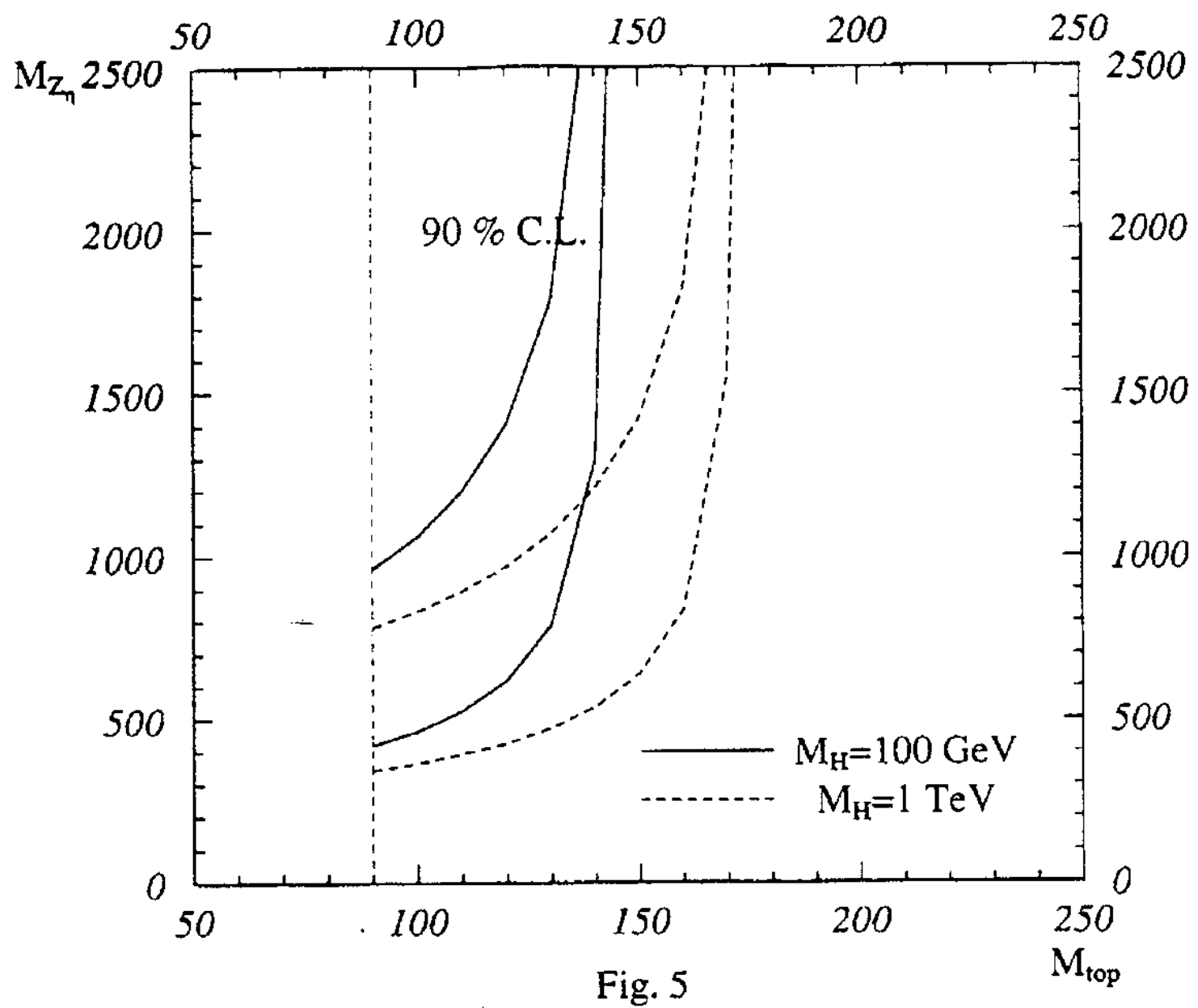

